\title{
Curvas de Tensión y Energía en Bandas Elásticas de Caucho Natural
}

\author{
Mario Bao, \\ Julio Archibold, \\ Nathaly Royer, \\ Jay Molino,
}

Departmento de Biociencias y Salud Pública,

Corregimiento de Ancón, Albrook, Paseo Diógenes de la Rosa,

Universidad Especializada de las Américas, Panamá

Doi:10.19044/esj.2019.v15n33p90 ～URL:http://dx.doi.org/10.19044/esj.2019.v15n33p90

\section{Resumen}

Las bandas elásticas son frecuentemente usadas para terapias y entrenamiento resistivo sin considerar su comportamiento mecánico y esto puede causar lesiones. Adicional a esto, dada las propiedades visco elásticas del caucho, se deben tomar ciertas consideraciones puesto que ante estreses cíclicos las curvas de tensión varían y por ende la fuerza que el paciente ejerce para estirar la banda. El objetivo de este estudio es la caracterización mecánica de las bandas de caucho natural comercial. Para ello se realizaron pruebas de tensión al material donde se determinó la zona elástica y el efecto de estreses cíclicos en las curvas características del material (en la zona elástica). Se encontró que el caucho natural presenta un comportamiento lineal (hook) a muy bajos estreses. La tensión de rotura para el material fue determinada experimentalmente.

Palabras claves: Estrés en bandas elásticas, Energía, Terapias con bandas, Fuerza, Resistencia 


\title{
Tension and Energy Curves in Natural Rubber Elastic Bands
}

\author{
Mario Bao, \\ Julio Archibold, \\ Nathaly Royer, \\ Jay Molino, \\ Departmento de Biociencias y Salud Pública, \\ Corregimiento de Ancón, Albrook, Paseo Diógenes de la Rosa, \\ Universidad Especializada de las Américas, Panamá
}

\begin{abstract}
Elastic bands are often used for therapies and resistive training without considering their mechanical behavior and this can cause injuries. In addition to this, given the viscoelastic properties of rubber, certain considerations must be taken since in the case of cyclic stresses the tension curves vary and therefore the force that the patient exerts to stretch the band. The objective of this study is the mechanical characterization of commercial natural rubber bands. For this, stress tests were carried out on the material where the elastic zone and the effect of cyclic stresses on the characteristic curves of the material (in the elastic zone) were determined. It was found that natural rubber exhibits a linear behavior (Hook) at very low stresses. The ultimate tensile stress for the material was determined experimentally.
\end{abstract}

Keywords: Stress in elastic bands, Energy, Band therapies, Strength, Resistance

\section{Introduction}

Las bandas elásticas son ampliamente utilizadas en programas terapéuticos para que los pacientes recuperen movilidad a través de ejercicios de tensión modificable y las misma se han convertido en herramientas importante para la salud, sobre todo en pacientes de edad avanzada (Álvarez \& Zapata, 2008; Benito, Cupeiro, \& Calderón, 2008; Mikesky, Topp, Wigglesworth, Harsha, \& Edwards, 1994; Simoneau, Bereda, Sobush, \& Starsky, 2001). Se usa caucho natural para estos propósitos puesto que es menos susceptible a rotura dados los estreses por las magnitudes que son estirados y contraídos durante la terapia. Adicional a esto, al ser u material asequible, son fáciles de utilizar y no requieren de un mantenimiento 
específico.Todos los materiales que se alargan de forma directamente proporcional a la fuerza que produce la deformación obedecen a la ley de Hooke. Cuando deja de actuar la fuerza que produce la deformación, si está en la región elástica, un material recupera su forma original. Sin embargo, el comportamiento bajo tensión de una banda de caucho, a la que normalmente nos referimos como bandas elásticas, estrictamente, no corresponde a una elongación lineal. Cuando el material el estirado y luego le permite regresar a su forma original, se observa una histéresis. La misma es una indicación de que parte del trabajo mecánico se pierde durante el ciclo de estiramiento y contracción de la banda elástica. Esto es una característica del caucho natural y la misma debe ser considerada en las terapias (Benito, Cupeiro, \& Calderón, 2008; Guisasola, Carratalá, Calduch, \& Lucas, 2016; Inez, Pereira, \& Chagas, 2007; Reinehr, Carpes, \& Mota, 2008).

Existen varios cauchos que se pueden encontrar en el comercio. Los mismos poseen diferentes módulos de elasticidad lo que provee diferentes tipos de resistencia dependiendo de la terapia (Carbone, Pierro, \& Recchia, 2015; Mullins \& Tobin, 1966; Triana \& Fajardo, 2012).Sin embargo, es imperativo para el terapeuta entender el comportamiento mecánico del material, debido a que el uso excesivo de estas herramientas puede deteriorar la calidad de vida del paciente o inclusive alargar el tiempo de recobro de este. Como bien lo establece Pereira et al., si se emplea una fuerza inadecuada (e.g. excesiva), torque o mucha presión, esto puede causar inflamación, cicatrices queloides o inclusive deformidades.

El objetivo de este estudio se enfocará en determinar la tensión de rotura, las curvas de tensión (de color crema y forma plana), el ciclo de histéresis, y la energía liberada por una banda elástica comercial (cuando la misma se mantiene estirada por un intervalo de tiempo determinado). (Allen, 2019; DeBever, 1992; Frunza \& Diaconescu, 2006; Treloar, 1973).

\section{Marco Metodológico}

\section{a. Materiales:}

En la elaboración de las pruebas se utilizaron bandas elásticas planas, con un ancho de $1.90 \mathrm{~cm}$ y un grosor de $0.10 \mathrm{~cm}$; muestras similares se usaron de bandas elásticas tubulares de color crema. Ambas bandas están hechas de caucho natural via vulcanización con un peso molecular en el rango de los 300kDa. La única diferencia entre ambas es la pigmentación y forma física de la misma.

Los primeros dos estudios se realizaron usando un equipo de medición de tensión diseñado en la universidad. El mismo consiste en dos prensas que sujetan el material y luego se aplica una fuerza para alargar la muestra. Se emplearon masas graduadas y una cinta métrica en milímetros para registrar la elongación de la muestra de caucho. 
Para determinar la tensión de rotura del material se utilizó una banda de cada tipo, con una longitud aproximada de $0.10 \mathrm{~cm}$. La prueba de histéresis se realizó para un total de 10 muestras de bandas elásticas de caucho tubulares y planas con longitudes de $5.00 \mathrm{~cm}$. Se empleó un calibrador vernier para adecuar las muestras a longitudes determinados y se utilizaron también, masas calibradas para controlar las fuerzas ejercidas para estirar las bandas.

El segundo estudio se realizó utilizando un dispositivo para disparar los proyectiles, diseñado en la universidad. El mismo se compone de dos prensas, que sujetan los dos extremos de la banda, fijándolo en posiciones exactas sobre una plataforma de madera. Se utilizó un proyectil con una masa de $20 \mathrm{~g}$. El mismo fue pintado de forma que, al impactar con una plancha de hielo seco colocado sobre el suelo, dejaba una marca. Con una cinta marcada en milímetros se midió la distancia recorrida por el proyectil. El experimento se realizó para un total de 10 muestras. Para este estudio hay dos variantes. La primera consiste en estirar la banda y dejarla estirada por 3 segundos antes de soltarla cargada con el proyectil, en la segunda modalidad se mantuvo la banda estirada por 5 segundos, antes de soltarla con el proyectil. El tiempo se midió con un cronómetro.

\section{b. Procedimientos:}

Se emplearon los siguientes procedimientos experimentales:

Procedimiento A (Prueba de Estrés): La temperatura del experimento se mantuvo $20^{\circ} \mathrm{C}$. Un esquemático del aparato empleado se muestra en la Figura 1.

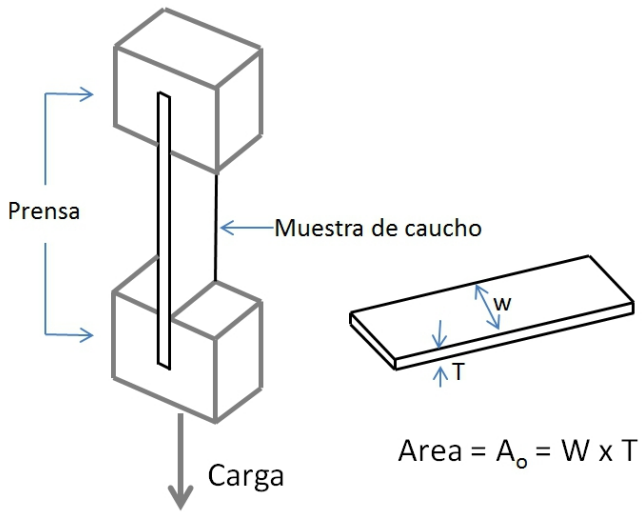

Figura 1: Esquemático del dispositivo en el Procedimiento A.

Con el calibrador vernier, el espesor de la muestra de caucho fue medido de manera tal que el área de sección transversal, $\mathrm{A}_{\mathrm{o}}$, pudo ser calculada. El material es colocado entre las prensas y seguidamente se procede a marcar los extremos de la tira de la muestra que quedaron entre las prensas. La parte superior de la prensa está fijada mientras que la parte de abajo posee un arnés que permite colocar masas calibradas que son usadas para aumentar la tensión en la liga. Estas masas calibradas fueron añadidas progresivamente. 
Atrás de la muestra se encuentra una cinta métrica milimetrada. Luego de añadir cada masa se permite que el caucho repose por unos treinta segundos dado a que el proceso de estiramiento es de carácter entrópico y 30 segundos demostraron ser suficientes para que el caucho alcanzara la temperatura del ambiente. Mientras se enfría el caucho, se procede a tomar las mediciones de longitud de este tras cada adición de masa.

Una vez que los datos son recolectados se grafica una curva tensióndeformación, donde se busca el alargamiento del material en función del estrés aplicado por las masas. El estrés ingenieril se calcula a través de la ecuación (1) $\boldsymbol{\sigma}=\boldsymbol{P}$ ? $\boldsymbol{A}_{\mathbf{0}} \quad$, donde $\sigma$ es el estrés ingenieril y $\mathrm{P}$ es el peso aplicado a la banda.

$$
\sigma=\frac{P}{A_{0}}
$$

Para determinar el alargamiento, $\varepsilon$, se utiliza la ecuación (2).

$$
\epsilon=\frac{\Delta L}{L_{o}} \times 100
$$

Donde $\Delta \mathrm{L}$ es el cambio de longitud del caucho luego de haber aplicado una carga $\mathrm{y}_{\mathrm{o}}$ es la longitud inicial de la muestra. Se multiplica por un factor de 100 para denotar porcentaje.

Para calcular la tensión de rotura, se añaden masas hasta que el material seda y sufra una rotura, mientras que para determinar los ciclos de histéresis solo se carga y descarga la muestra en un área donde se tiene certeza que no había deformaciones plásticas. Luego de varios experimentos, se determinó que dicha región era a $30 \%$ de elongación de la longitud original de la muestra.

Procedimiento B (Prueba de Energía): La banda elástica es colocada sobre dos prensas fijas. Las bandas son estiradas y se ancla a un disparador. El dispositivo diseñado asemeja a una bayoneta. Seguidamente se coloca el proyectil sobre el material. En el otro extremo hay una lámina de hielo seco. Después de cada tiro, se mide la altura a la que golpea el proyectil la plancha. No hay variaciones en la altura a la que se lanza el proyectil ni la distancia de separación entre el dispositivo y la plancha de hielo seco. En todos los experimentos las bandas fueron estiradas la misma longitud.

Una vez que se hayan recolectado los datos, se calcula el tiempo de caída a través de la ecuación (3) para caída libre,

$$
\boldsymbol{t}=\sqrt{\frac{2 \cdot \Delta \boldsymbol{h}}{g}}
$$

donde $t$ es el tiempo, recorrido del proyectil, sobre el aire; $\Delta h$ la altura de caída y $g$ la aceleración por la gravedad. Como el proyectil fue lanzado sin velocidad vertical $\left(V_{y}\right)$, para determinar la energía total se necesita saber la magnitud de la misma considerando que existe una velocidad inical $V_{x}$. No se se considera la resistencia del aire en el análisis. Conociendo el tiempo de caída se puede calcular la velocidad atendiendo que la distancia horizontal entre el proyectil 
y el punto de impacto fue medida. Siendo $V$ la magnitud de la velocidad, la energía total del proyectil al momento del impacto está dada por la ecuación (4):

$$
E=\frac{m \cdot V^{2}}{2}+\boldsymbol{m g} \Delta \boldsymbol{h}
$$

Donde $m$ es la masa del proyectil y $V$ es la magnitud de la velocidad total.

Este experimento se repitió 10 veces para cada tipo de banda elástica, utilizando el mismo proyectil. Donde luego se promedia la energía liberada; en situaciones donde la banda es estirada y mantenida por 3 y 5 segundos, antes de ser disparada.

\section{Resultados y Discusión}

\section{a. Tensión de Rotura}

La Figura 2 muestra la tensión de rotura para una banda plana (izquierda) y una tubular (derecha). Nótese que ambas bandas de caucho poseen una región hookeana ante bajos estreses (aproximadamente un $30 \%$ de elongación), luego aparece una región no lineal antes de alcanzar una elongación del 100\%. Seguidamente ambas muestras poseen un comportamiento hookeano y en el caso especial de la banda tubular, cuando la misma alcanza una elongación del $400 \%$, se observa que la constante de proporcionalidad aumenta, sin embargo, sigue manifestando un comportamiento hookeano. Esto indica que las bandas, a pesar de estar hechas del mismo material poseen una estructura molecular diferente. Las primeras tres regiones son características de un comportamiento elástico no linear y la entropía del arreglo molecular puede ser considerada como la suma de la entropía de las cadenas de polímeros independientes. Por eso se pueden diferencias 3 regiones con claridad en el caso de las bandas tubulares.
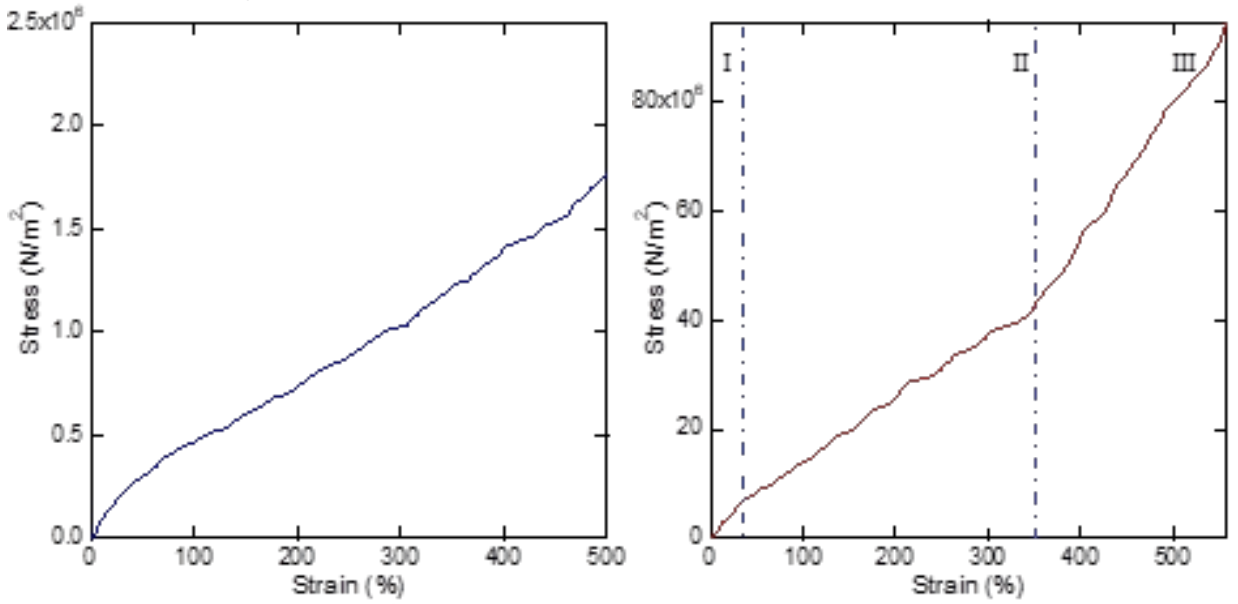

Figura 2: Curva de estrés vs tensión para (izquierda) una banda de caucho natural plano y (derecha) una banda de caucho natural con forma tubular. 
b. Ciclo de Histéresis:

La Figura 3 muestra el ciclo de histéresis en la banda tubular y una plana. Nótese que la banda tubular tiene un ciclo de histéresis diferente a la plana. Dado a que poseen coeficientes de elasticidad diferente, se necesita más peso para que la banda pueda elongarse. Cuando esto ocurre la elongación es escalonada y no gradual como se aprecia en la banda plana. Esto es una limitación del proceso experimental. Sin embargo, a pesar de la forma irregular que posee el ciclo de histéresis de la banda tubular (no se aprecia un ciclo propiamente), cabe destacar que es evidente que la curva de carga es menor que la de descarga, indicativo de que energía se pierde en el proceso. Esta energía se manifiesta en la forma de calor que es disipado a la atmósfera. No se midió el aumento de temperatura que tuvieron las bandas al ser elongadas sin embargo cualitativamente, se identificó un aumento de temperatura en las superficies de las bandas. Adicional a esto, las bandas jamás regresaron a su longitud original. Este resultado se debe a la reestructuración molecular del material, dado que, al ejercer una fuerza, la configuración del polímero varía, es decir, se elonga y no puede restituirse al estado original.
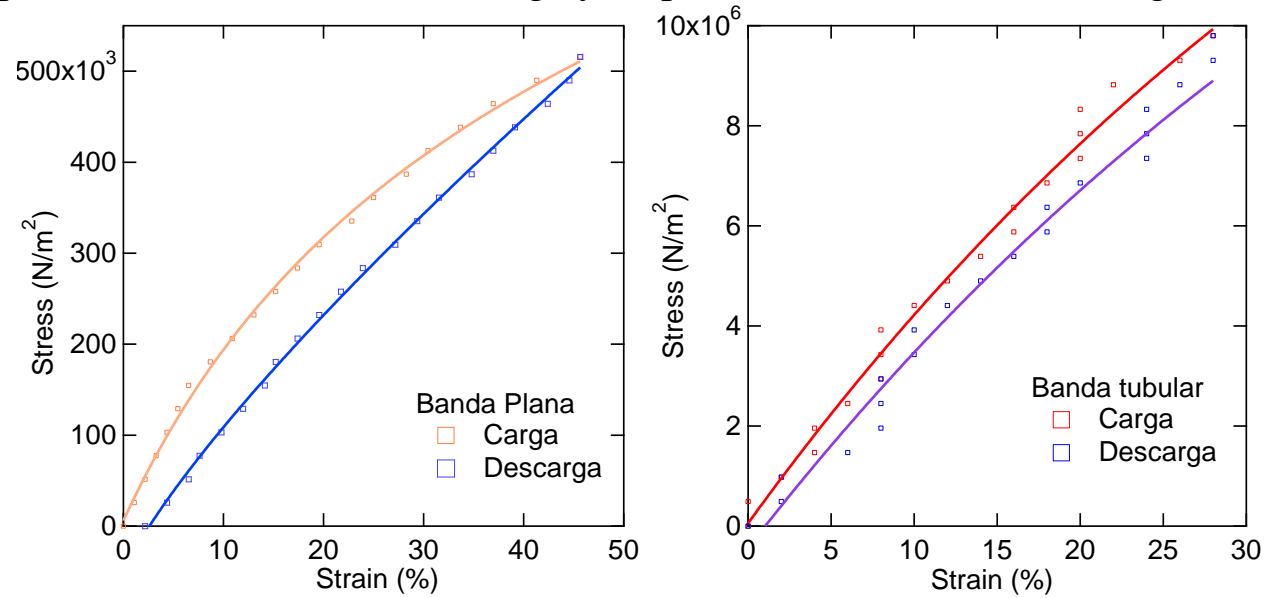

Figura 3: Ciclo de histéresis de: (izquierda) una banda Plana; (derecha) banda tubular.

También se evaluó las curvas de estrés y tensión para varios ciclos en la banda plana. Los resultados son mostrados en la Figura 4. Se registraron 3 ciclos y no hubo variación apreciable entre cada ciclo. Indicando que la banda se encontraba en equilibrio después del primer ciclo. Los resultados, cuando se comparan con los de la Figura 4, son indicativos de una condición de preestrés en el material que debió de ser considerada en el protocolo de medición. 


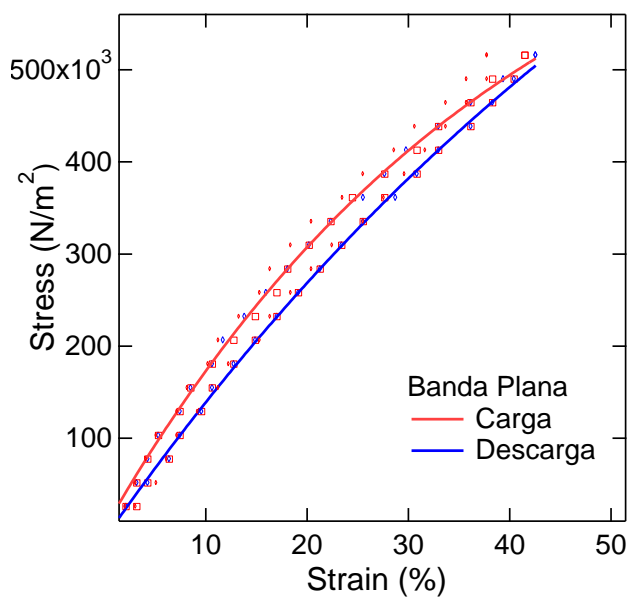

Figura 4: Comparación de los niveles de estrés para ciclos de carga y descarga repetitivos para la banda plana.

Dado a que los ciclos repetidos de deformación afectan la resistencia del caucho haciendo que requiera menos fuerza para estirar la banda, efecto Mullins, la energía en la banda elástica disminuye porque hay una ruptura y formación de nuevos enlaces durante las deformaciones. Las moléculas se alinean en la dirección de la fuerza aplicada (Inez, Pereira, \& Chagas, 2007; Mullins \& Tobin, 1966; Price \& Wolf, 1976).

\section{c. Energía:}

La Tabla 1 se demuestra que, al mantener la banda elástica estirada por más tiempo, la energía total liberada (aquella que se emplea para el disparo del proyectil) es menor. Es decir, que la energía liberada tiene una relación inversamente proporcional al tiempo que se mantiene estirado la banda. Esto implica que la entropía del mismo material decae con el pasar del tiempo, dado a la disipación de la energía con el ambiente. Lo cual conlleva a la utilización de una fuerza menor para mantener la banda estirada. Este fenómeno se aprecia también en las curvas de histéresis donde se le aplica una tensión al material elástico (carga) y después de un tiempo es liberado (descarga), de forma que regresan a su estado original. Donde la curva de carga requiere mayor energía que la mostrada durante descarga. Sin embargo, el experimento de energía no se realizó a una temperatura controlada, a diferencia de la prueba de histéresis, y esto afecta la precisión de los resultados; aunque, cualitativamente, encontramos evidencia de que al dejar la liga estirada por más tiempo resulta en una pérdida de energía mecánica que se sentía en la forma de calor. 
Tabla 1: Energía Promedio Liberado.

\begin{tabular}{l|llll} 
Material & \multicolumn{3}{c}{ Banda Tubular } & \multicolumn{2}{c}{ Banda Plana } \\
\hline$\Delta \mathrm{t}$ & $3 \mathrm{~s}$ & $5 \mathrm{~s}$ & $3 \mathrm{~s}$ & $5 \mathrm{~s}$ \\
Energía Liberada (J) & $0.0930 \mathrm{~J}$ & $0.0859 \mathrm{~J}$ & $0.1216 \mathrm{~J}$ & $0.1010 \mathrm{~J}$
\end{tabular}

Nótese que las bandas tubulares tienen una pérdida de energía menor que a la banda plana y esto se debe a que cada banda posee coeficientes de elasticidad diferentes y estructura molecular diferente. El efecto de la configuración molecular en las propiedades mecánicas es parte de la investigación que se está realizando.

\section{Conclusiones}

Los resultados demuestran que las bandas poseen varias regiones donde se comportan de manera lineal y no lineal. Considerando esto, es preciso que el terapeuta considere este comportamiento ya que el módulo de elasticidad en la tercera región es mayor y puede causar laceraciones en el paciente. Por lo tanto, es preferible que las terapias consideren una elongación máxima del $30 \%$ para asegurarse que las mismas tengan un comportamiento hookeano. De igual forma hay que considerar la velocidad a la que se hace la terapia. La resistencia de la banda elástica varía dependiendo de la velocidad con la que son estiradas y contraídas. Esto se debe a que esa energía mecánica se pierde en la forma de calor. Finalmente, al hacer las terapias, hay que considerar aspectos importantes como el material y geometría de las bandas para asegurar la terapia correcta para el paciente.

\section{Agradecimientos}

Esta investigación fue parcialmente apoyada por la Universidad Especializadas de las Américas a través del fondo 009-2017 y al grupo de "Udelistas Pro-Investigación" con sus asesorías sobre cómo redactar una investigación.

\section{References:}

1. Allen, D. J. (2019). Stress-Strain Characteristics of Rubber-Like Mateihals : Experwent and Analysis.

2. Álvarez, Á., \& Zapata, R. (2008). Las Bandas Elásticas, Un Medio Par El Mejoramiento De La Fuerza Muscular En Los Adultos Mayores, 181.

3. Benito, P., Cupeiro, R., \& Calderón, F. (2008). Ejercicio físico como terapia no farmacológica en la artrosis de rodilla. Reumatología Clínica, 6(3), 153-160. https://doi.org/10.1016/j.reuma.2008.11.021

4. Carbone, G., Pierro, E., \& Recchia, G. (2015). Loading-unloading hysteresis loop of randomly rough adhesive contacts. Physical Review 
E - Statistical, Nonlinear, and Soft Matter Physics, 92(6). https://doi.org/10.1103/PhysRevE.92.062404

5. DeBever, A. (1992). Dynamic behavior of rubber and rubber like materials Literature survey Dynamic behavior of rubber and rubber like materials (First). Eindhoven University of Technology.

6. Frunza, G., \& Diaconescu, E. (2006). Hysterisis and Mechanical Fatigue. THE ANNALS OF UNIVERSITY "DUNĂREA DE JOS “ OF GALAŢI FASCICLE VIII, 61-66.

7. Guisasola, E., Carratalá, V., Calduch, F., \& Lucas, F. (2016). El papel de la rehabilitación tras las reparaciones de las inestabilidades de muñeca. Revista Iberoamericana de Cirugía de La Mano, 44(2), 131142. https://doi.org/10.1016/j.ricma.2016.09.001

8. Inez, M., Pereira, R., \& Chagas, S. (2007). Efeito do treinamento contra-resistência isotônico com duas velocidades de movimento sobre os ganhos de força, 13(21), 91-96.

9. Mikesky, A. E., Topp, R., Wigglesworth, J. K., Harsha, D. M., \& Edwards, J. E. (1994). Efficacy of a home-based training program for older adults using elastic tubing. European Journal of Applied Physiology and Occupational Physiology, 69, 316-320. https://doi.org/10.1007/BF00392037

10. Mullins, L., \& Tobin, N. (1966). Stress Softening in rubber vulcanizates. Part I. Use of a Strain Amplification Factor to Describe Elastic Behavior of Filler-Reinforced Vulcanized Rubber. Journal of Applied Polymer Science, 9(9), 2993-3009.

11. Price, C., \& Wolf, F. (1976). Thermodynamics of Rubber Elasticity [and Discussion]. Proceedings of the Royal Society of London. Series A, MAthematica and Physical Sciences, 351(1666), 331-350.

12. Reinehr, F., Carpes, F., \& Mota, C. (2008). Influence of core stabilization training on low back pain and stability. Fisioter. Mov., 21(1), 123-129.

13. Simoneau, G. G., Bereda, S. M., Sobush, D. C., \& Starsky, A. J. (2001). Biomechanics of Elastic Resistance in Therapeutic Exercise Programs. Journal of Orthopaedic \& Sports Physical Therapy, 31(1), 16-24. https://doi.org/10.2519/jospt.2001.31.1.16

14. Treloar, L. (1973). The elasticity and related properties of Rubbers. Reports on Progress in Physics, (36), 625-696.

15. Triana, C. A., \& Fajardo, F. (2012). Dependence of some mechanical properties of elastic bands on the length and load time. European Journal of Physics, 33, 771-784. https://doi.org/10.1088/01430807/33/4/771 